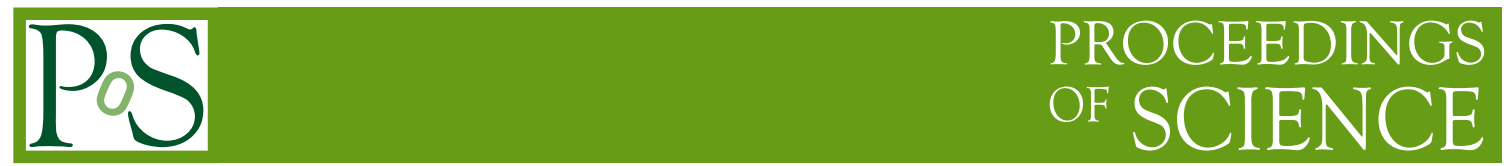

\title{
Experimental perspectives on next generation EDM searches
}

\author{
Wolfgang Korsch* \\ Department of Physics and Astronomy, University of Kentucky, Lexington, KY 40506 \\ E-mail: korschepa.uky.edu
}

\begin{abstract}
The search for permanent electric dipole moments in non-degenerate systems has become a very active field of research in recent years. The experimental sensitivity has reached limits which pose severe constraints on physics beyond the Standard Model. This talk will review and summarize some of the ongoing efforts.
\end{abstract}

The 7th International Workshop on Chiral Dynamics,

August 6 -10, 2012

Jefferson Lab, Newport News, Virginia, USA

${ }^{*}$ Speaker. 


\section{Introduction}

The search for permanent electric dipole moments (EDM) in nucleons, atoms, molecules, or other fundamental particles has been an ongoing experimental effort for more than five decades. A non-zero EDM simply implies that electric charge is not uniformly distributed "inside" such a particle, giving rise to the violation of time-reversal symmetry. Already in 1950 E. M. Purcell and N. F. Ramsey argued that a non-zero EDM could arise due to parity violation [1]. It is easy to comprehend this argument by studying the interaction Hamiltonian between external magnetic and electric fields and a particle with spin s:

$$
H=-(\mu \cdot \mathbf{B}+\mathbf{d} \cdot \mathbf{E})=-\left(d_{M} \frac{\mathbf{s}}{|\mathbf{s}|} \cdot \mathbf{B}+d_{E} \frac{\mathbf{s}}{|\mathbf{s}|} \cdot \mathbf{E}\right) .
$$

Here $\mu$ and $\mathbf{d}$ denote the magnetic and electric dipole moments of the particle. It is worth emphasizing that $\mathbf{d}$ has to be aligned along the direction of the particle's spin. While the magnetic field is even under parity transformation, the electric field is not, so a finite value of $\mathbf{d}$ (or $d_{E}$ ) implies that $H$ does not conserve parity. In more recent years the concept of parity violation has been expanded to the "CPT Theorem". It is believed, even beyond the Standard Model of particle physics, that nature is invariant under a combined symmetry operation consisting of charge conjugation $(C)$ and parity transformation $(P)$ and time reversal $(T)$. Indeed, the Hamiltonian in Eq. 1.1 does violate time reversal, and hence CP symmetry, if $|\mathbf{d}| \neq 0$. In our discussion, we assume that no degenerate states exist in the system of interest.

Present limits on EDMs pose already stringent constraints on many "new physics" models. See reference [2] for a current update. Some recent experimental results, together with the Standard Model (CKM) predictions, are listed in table 1. The discovery of a finite EDM at present experimental sensitivities would unambiguously reveal physics beyond the Standard Model of particle physics.

Table 1: Recent experimental limits on permanent EDMs (incomplete list).

\begin{tabular}{lccc}
\hline Particle & Limit $[e \cdot \mathrm{cm}](\geq 90 \%$ C.L. $)$ & Experiment & SM Prediction $[e \cdot \mathrm{cm}]$ \\
$e^{-}$ & $10.5 \times 10^{-28}$ & Hinds et al. $[3]$ & $<10^{-38}[5]$ \\
$\mu$ & $1.8 \times 10^{-19}$ & Bennett et al. $[4]$ & $<10^{-36}[6]$ \\
$n$ & $2.9 \times 10^{-26}$ & Baker et al. $[10]$ & $<10^{-32}[7]$ \\
${ }^{199} \mathrm{Hg}$ & $3.1 \times 10^{-29}$ & Griffith et al. $[12]$ & $\sim 10^{-33}[9]$ \\
${ }^{129} \mathrm{Xe}$ & $3.4 \times 10^{-27}$ & Rosenberry et al. $[11]$ & $\sim 10^{-34}[8]$ \\
${ }^{205} \mathrm{Tl}$ & $9.4 \times 10^{-25}$ & Regan et al. $[13]$ &
\end{tabular}

A non-zero permanent EDM has numerous and far reaching implications, e.g. it could, at least partially, explain the baryon-anti-baryon asymmetry of the universe and it would reveal new sources of $C P$ violation.

In the following sections we describe shortly some ongoing efforts. Section 2 focuses on neutron EDM searches, Section 3 summarizes the activities on atomic and molecular systems, and Section 4 describes some recent ideas on how to access EDMs of light ions. 


\section{Neutron EDM searches}

The first dedicated neutron EDM measurement dates back to 1957 when J. H. Smith, E. M. Purcell, and N. F. Ramsey published their result of an experiment that was performed at ORNL using a magnetic resonance technique [14]. An upper bound of $(0.1 \pm 2.4) \times 10^{-20} \mathrm{e} \cdot \mathrm{cm}$ was reported for the magnitude of the EDM. This was just the beginning of still ongoing efforts to find violation of fundamental time-reversal symmetry. During the past fifty years the limit was improved continuously at a rate of roughly one order of magnitude per decade. Basically all neutron EDM searches utilized magnetic resonance techniques and, since about 1980, ultra-cold neutrons (UCNs) [15] were used instead of thermal or cold neutron beams. The best result thus far was obtained by the Sussex/RAL/ILL collaboration at the ILL in Grenoble [10]. Their experimental procedure can be summarized as follows: First, longitudinally polarized UCNs were injected into a trapping volume $(\approx 211)$. Inside the trap the neutrons were exposed to a weak uniform static magnetic field ( $\approx 10$ mGauss) which was superimposed by a parallel or anti-parallel electric field with a magnitude of $10 \mathrm{kV} / \mathrm{cm}$. After injection, an oscillating magnetic field was applied which rotated the neutron spins perpendicular to the static fields. A free spin precession followed for about $130 \mathrm{~s}$. Then a second pulse was applied to rotate the neutron spins back to their original directions. Finally, the neutron polarization was measured to determine whether the electric field influenced the precession frequency. This technique is known as the Ramsey separated-oscillatoryfield magnetic resonance method and is common to several modern EDM searches. The final result reported by the collaboration constrains $d_{n}$ to less than $2.9 \times 10^{-26} e \cdot \mathrm{cm}$ [10].

A new generation of EDM experiments has emerged with the goal to improve the present limit by two orders of magnitude. Novel developments are either in progress or have been proposed at the PSI in Switzerland [18], at the ILL in France [18], at the FRM-2 in Germany [18], at the SNS in the US [18], at RCNP/TRIUMF in Japan/Canada [18]. In the following section the planned EDM search at the SNS will be described in more detail.

\subsection{The SNS experiment}

Inspection of the interaction Hamiltonian, Eq. 1.1, suggests that $d_{n}$ can be extracted by monitoring the change in spin precession frequency of the neutron when the electric field, $\mathbf{E}$, is reversed relative to the magnetic field, B. Solving Eq. 1.1 for $d_{n}$ yields:

$$
d_{n}=\frac{h \Delta v}{4 E},
$$

where $h$ is the Planck constant, $\Delta v$ is the change in precession frequency when $\mathbf{E}$ is reversed relative to $\mathbf{B}$, and $E$ is the magnitude of the electric field. The statistical uncertainty on $\delta d_{n}$ is bounded by the uncertainty principle:

$$
\delta d_{n}=\frac{\hbar}{4 E T_{m} \sqrt{m N}},
$$

where $T_{m}$ is the time to perform a neutron spin precession measurement, and $m$ denotes the number of separate complete measurements with $N$ neutrons. It is obvious from this formula that long neutron spin precession times, many measurement cycles, and a large number of neutrons are needed to achieve a minimum statistical uncertainty. 
The newly constructed Spallation-Neutron-Source (SNS) at the Oak Ridge National Laboratory which commenced operation in 2006 appears to be an ideal environment to conduct a high precision neutron EDM experiment. A $1.4 \mathrm{MW}$ pulsed proton beam $\left(\mathrm{E}_{p}=1.0 \mathrm{GeV}\right)$ impinges on a high power mercury target and spallation neutrons are generated with unprecedented (pulsed) intensity. After moderation the neutrons have a typical temperature of $T_{n} \sim 20 \mathrm{~K}$. In 1975, R. Golub and R. Pendlebury pointed out that the local density of free neutrons can be increased significantly when they are converted to ultra-cold neutrons [15]. Specifically, neutrons with a wavelength of 8.9 $\AA$ can be down-scattered to milli-Kelvin temperatures via single-phonon excitations in superfluid helium-4 [16]. This concept can be exploited by capturing and storing such UCNs in a container.

The SNS cold neutron beam will be used in combination with a system of velocity filters (choppers) to generate $8.9 \AA$ neutrons with a narrow bandwidth. These neutrons will then be transported and polarized using standard super-mirror techniques, and finally they will be injected into two target cells containing superfluid helium-4. Any neutron that experiences a conversion to a UCN will has a chance to be trapped. The whole target region will be exposed to a superposition of a very uniform small magnetic field $(\approx 30 \mathrm{mG}$ ) and a strong electric field (up to $50 \mathrm{kV} / \mathrm{cm}$ ). The main challenge of the experiment is to measure the precession frequency of the neutrons very precisely. To accomplish this goal it is planned to take advantage of the strong spin dependence in the reaction: $\vec{n}+{ }^{3} \overrightarrow{H e}^{++} \rightarrow t+p+764 \mathrm{keV}$. For that purpose, a small amount of polarized ${ }^{3} \mathrm{He}$ will be injected into the helium- 4 cell as well. Both the neutrons and ${ }^{3} \mathrm{He}$ nuclei will be prepared in such a way that all spins are aligned along the same direction (perpendicular to the magnetic field) at the beginning of a measurement cycle. Due to the difference in the magnetic moments of the two species, their spins will precess at different rates and therefore the rate at which the above reaction occurs is modulated accordingly. The protons released in this process carry enough energy to generate scintillation light in the superfluid medium. The scintillation light is detected and it is used as a measure of the relative spin precession frequency. A non-zero neutron EDM will manifest itself in a correlation between the light modulation and the direction of the electric field. Note that the effect of an external electric field on the precession of the helium-3 spins is vastly reduced due to the shielding effect of the atomic electrons. We also intend to monitor the precession frequency of the ${ }^{3} \mathrm{He}$ spins independently (using SQUIDs). This frequency information will help to keep track of the stability of the magnetic field.

The experimental techniques described in this paper appear to be promising to overcome statistical limitations experienced in previous endeavors to improve the electric-dipole-moment of the neutron. Given a realistic funding scenario data taking is expected to start around 2017. A more complete description of the whole experiment can be found in Ref. [17].

\section{EDM searches in atomic and molecular systems}

The apparent sensitivity to physics beyond the Standard Model has revived a large number EDM searches on atomic systems as well. Current searches of time-reversal violation in atomic systems can be split into two categories: highly polar diatomic molecular systems and diamagnetic atoms with, ideally, highly deformed nuclei. Polar molecules with unpaired electrons exhibit an increased sensitivity to the electron EDM since the bound electron is preferentially located along the internuclear axis. The magnitudes of the internal electric fields can reach tens of gigavolts per 


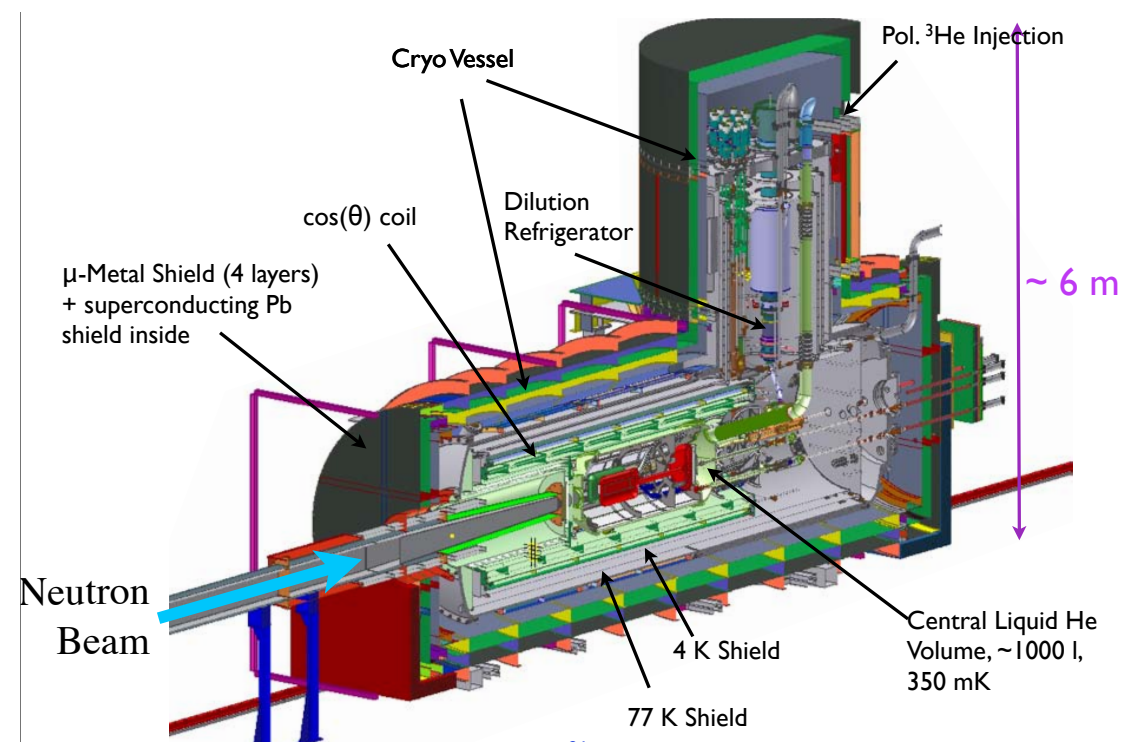

Figure 1: Design concept of the SNS-EDM apparatus.

centimeter. Molecules such as $\mathrm{HfH}^{+}, \mathrm{PbF}, \mathrm{PbO}, \mathrm{ThO}, \mathrm{WC}$, and $\mathrm{YbF}$ serve as possible candidates. The enhancement of $P$ - and $T$-odd effects can be explained by the small splitting of energy levels with opposite parity. The best limit on $d_{e}$ to date is $10.5 \times 10^{-28} e \cdot \mathrm{cm}$ and was extracted from studies on $\mathrm{YbF}[3]$.

\section{EDM searches in light ion systems}

During the last decade several proposals related to the search of light ion EDMs have been developed. The basic idea is to store polarized light ions, e.g. protons or deuterons, in a storage ring and monitor the spin precession carefully. The concept can be summarized by means of the Thomas-BMT formula which describes the motion of charged particle spins in the presence of electromagnetic fields. The equation can be expressed in the form [19]:

$$
\vec{\Omega}=-\frac{q}{m_{0}}\left\{G \vec{B}+\left(\frac{1}{\gamma^{2}-1}-G\right)\left(\frac{\vec{\beta} \times \vec{E}}{c}\right)+d_{i} \frac{m_{0} c}{q \hbar|\mathbf{s}|}\left(\frac{\vec{E}}{c}+\vec{\beta} \times \vec{B}\right)\right\}
$$

where $\vec{E}$ and $\vec{B}$ are the electric and magnetic fields, perpendicular to the motion of the particles, in the laboratory frame. $G=(g-2) / 2$ is the anomalous magnetic moment and $d_{i}$ is the electric dipole moment of the particle. Certain choices of particle energy and electric and/or magnetic fields will eliminate any spin rotation relative to the momentum of the particles, i.e. the precession of the spins is "frozen". Therefore, by monitoring the polarization of the stored beam with high precision, 
stringent limits can be placed on the EDMs of the charged particles since the direction of the spins will not change in the absence of an EDM. Two collaborations, one in the United States [20] and one in Germany [21], are pursuing these ideas with the goal of measuring the EDMs of the proton and the deuteron at the $10^{-29} e \cdot \mathrm{cm}$ level.

\section{Acknowledgments}

This work is supported by the U.S. Department of Energy (DOE), Office of Nuclear Physics, under contract DE-FG02-99ER41101.

\section{References}

[1] E. .M. Purcell and N. F. Ramsey, Phys. Rev. 78807 (1950).

[2] M. Le Dall and A. Ritz, CP-violation and electric dipole moments, in proceedings of The $5^{\text {th }}$ International Symposium on Symmetries in Subatomic Physics (2013).

[3] J. J. Hudson et al., Nature 473493 (2011).

[4] G. W. Bennett et al., Phys. Rev. D 80052008 (2009).

[5] I. B. Khriplovich and M. E. Pospelov Sov. J. Nucl. Phys. 53638 (1991).

[6] F. J. M. Farley et al. Phys. Rev. Lett. 93052001 (2004).

[7] I. B. Khriplovich and A. R. Zhitnitsky Phys. Lett. B 109490 (1982).

[8] M. J. Ramsey-Musolf and S. Su, Low energy precision tests of supersymmetry hep-ph/0612057v1 (2006).

[9] M. E. Pospelov and A. Ritz, Annals Phys. 318119 (2005).

[10] C. A. Baker et al., Phys. Rev. Lett. 97131801 (2006).

[11] M. A. Rosenberry and T. E. Chupp, Phys. Rev. Lett. 8622 (2001).

[12] W. C. Griffith et al., Phys. Rev. Lett. 102101601 (2009).

[13] C. B. Regan et al., Phys. Rev. Lett. 88071805 (2002)

[14] J. H. Smith, E. M. Purcell, and N. F. Ramsey, Phys. Rev. 108120 (1957).

[15] R. Golub and J. M. Pendlebury, Phys. Lett. 53A 133 (1975).

[16] R. Golub and J. M. Pendlebury, Phys. Lett. 62A 337 (1977).

[17] R. Golub and S. R. Lamoreaux, Phys. Rep. 2371 (1994).

[18] see http://nedm.web.psi.ch/ (PSI), https://www.neutronedm.org/index.html (ILL), http://http://nedm.ph.tum.de/ (Munich), http://www.phy.ornl.gov/nedm/ (SNS), http://fnp.kek.jp/English/activity_e.html (RCNP/TRIMUF).

[19] A. Lehrach, Project Overview and Computational Needs to Measure Electric Dipole Moments at Storage Rings, in proceedings of ICAP2012, 7 (2012).

[20] Storage Ring EDM Collaboration, A Proposal to Measure the Proton Electric Dipole Moment with $10^{-29} e \cdot c m$ Sensitivity, Brookhaven National Laboratory (2011).

[21] JEDI Collaboration, Search for Permanent Electric Dipole Moments at COSY (2012). 